\title{
La violence domestique: un sujet qui concerne aussi la médecine
}

\section{Christine Romann}

Dr, membre du Comité central de la FMH, responsable du Département promotion de la santé et prévention

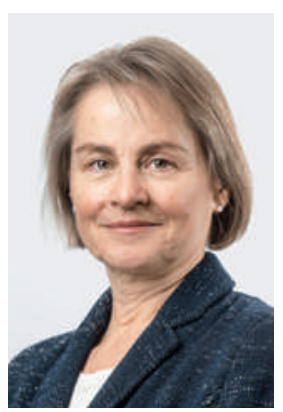

Que savons-nous, médecins, de la violence domestique? Face à des blessures ou des contusions, avonsnous le réflexe d'y penser dans le cadre de notre pratique quotidienne en cabinet ou aux urgences? La considérons-nous comme un phénomène marginal touchant principalement les migrants ou les patientes et patients issus d'un environnement social défavorisé?

Une telle approche - qui relève plutôt de préjugés - ne reflète nullement la réalité. En Suisse aussi, la violence domestique est un phénomène largement répandu qui touche les femmes de tous les milieux. L'environnement familial se révèle être le lieu de tous les dangers: c'est à la maison que les femmes sont le plus souvent victimes de violences. Le Bureau de l'égalité entre hommes et femmes fournit à ce sujet des statistiques éloquentes (www.egalite-suisse.ch). La violence vécue laisse des traces durables. L'éventail des symptômes recouvre les lésions corporelles directes en passant par les troubles psychosomatiques ou les pathologies psychiques comme les angoisses ou les dépressions, voire même une tendance au suicide. Pour les femmes concernées, les médecins sont souvent les premiers interlocuteurs. En règle générale, ces dernières ne rapportent pas d'emblée que leurs souffrances résultent d'une violence subie; un sentiment de honte mêlé à la peur les empêchant souvent de parler de ce qu'elles ont vécu. Parfois, les patientes ne sont même pas conscientes que leurs douleurs chroniques du bas-ventre ou leurs états d'anxiété sont le résultat de la situation traumatisante qui règne à la maison.

\section{La violence domestique touche toutes les couches de la société.}

Mais que faire lorsque la consultation ou l'examen médical soulève des soupçons indiquant que les problèmes de santé de la patiente - voire aussi d'un patient - pourraient être liés aux coups ou aux mauvais traitements infligés par le ou la partenaire? Et que faire lorsqu'à notre demande ou spontanément, la patiente nous raconte que son partenaire la maltraite?
La Conférence nationale «La violence domestique: un thème de santé publique», organisée en novembre dernier à Berne par le Bureau fédéral de l'égalité entre hommes et femmes, a tenté d'apporter des réponses à cette question et à d'autres. L'objectif de cette journée, destinée explicitement aux professionnels de la santé, a été de recueillir et de communiquer les connaissances acquises en matière de dépistage et de traitement de la violence domestique. Les différents projets présentés lors d'exposés ou d'ateliers ont encouragé la réflexion, permis d'évoquer des solutions, et dé-

\section{Les médecins sont appelés à aborder} systématiquement ce thème.

montré que plusieurs initiatives vont déjà dans la bonne direction. C'est ainsi que la doctoresse Barbara Bass, médecin-chef de la psychosomatique de la Clinique des femmes de la maternité Triemli, a présenté un modèle de dépistage et de soutien aux victimes de violence domestique à l'Hôpital zurichois du Triemli. Dans le présent numéro du Bulletin des médecins suisses, nous souhaitons sensibiliser au thème de la violence domestique et faire profiter des expériences acquises jusqu'à présent. Deux oratrices de la conférence, la doctoresse Sibil Tschudin, de Bâle, spécialiste en gynécologie et obstétrique, et la doctoresse MarieClaude Hofner, de l'Unité de médecine des violences du CHUV, Centre universitaire romand de médecine légale, à Lausanne, nous expliquent leurs activités respectives dans ce domaine délicat, à lire en pages 240 et 243. Le message principal de ces deux expertes est le suivant: les médecins doivent poser la question de la violence, systématiquement! De plus, ils doivent connaître les possibilités d'aide aux victimes de violence domestique, afin d'orienter rapidement et sans complications les femmes concernées. De cette manière, ces femmes éprouvent le sentiment d'être prises au sérieux, mais aussi que la violence domestique n'est pas une fatalité et ne peut en aucun cas être tolérée. 